\title{
Neuraltherapie - Definition, Wirkmechanismen und Wirksamkeit
}

Die zunehmend sowohl in Grundversorger- als auch in spezialisierten Praxen angewandte Neuraltherapie (als diagnostische und therapeutische Lokalanästhesie) ist auch aus keiner Schmerzklinik mehr wegzudenken. So wird sie in der Schweiz seit 2011 zur Schulmedizin gerechnet und ist Bestandteil der Grundversicherung.

1 n der Neuraltherapie werden Lokalanästhetika sowohl diagnostisch als auch therapeutisch eingesetzt. Die Methode nutzt die regulatorischen und plastischen Eigenschaften des Nervensystems. Eine wichtige Rolle spielt hierbei das autonome Nervensystem. Die selektive Reizlöschung durch das Lokalanästhetikum (,Reset“) an bestimmten Stellen beeinflusst die Organisation im Nervensystem sowie die (Mikro-)Zirkulation. Die Langzeitresultate bei chronischen Schmerzpatient*innen und bei Entzündungen lassen sich mit der Neurophysiologie erklären.

Entdeckt wurde die Neuraltherapie von den Gebrüdern Huneke 1925; Pioniere waren auch Leriche, Ricker, Speranski, Pischinger, Dosch, Bergsmann, Hopfer und andere..$^{1-4}$ Historisch wird eine lokale und segmentale Neuraltherapie von der übersegmentalen Störfeldtherapie unterschieden. Neue Erkenntnisse der Neuroanatomie und Neurophysiologie zeigen allerdings, dass Segmentgrenzen nicht eindeutig definiert werden können. So wird in der neueren Literatur das sogenannte „Störfeld“ auch als ,,neuromodulatorischer Trigger" ${ }^{5}$ beschrieben, dessen Auswirkungen sich sowohl innerhalb als auch außerhalb der metameren segmentalen Ordnung manifestieren können (siehe Abbildung). Potenzielle „Störfelder“ mit Einfluss auf das Immun- und Entzündungsgeschehen und auf nozizeptive Sensibilisierungsprozesse können zum Beispiel verlagerte Weisheitszähne, chronische Tonsillopharyngitiden, Narben usw. sein. ${ }^{1-4}$

\section{INJEKTIONSARTEN}

Entsprechend den reflektorischen Zusammenhängen (siehe Abbildung) ergeben sich folgende Injektionsmöglichkeiten, die individuell kombiniert werden können:

- Hautquaddeln

- Myofasziale Triggerpunkte

- Gelenke

- Sympathische Ganglien

- Periphere Nerven

- Narben

- usw.

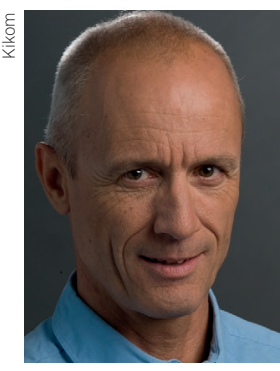

Von

Prof. Dr.

Lorenz Fischer

Facharzt Allg. Innere Medizin, em. Dozent

Neuraltherapie an der Universität Bern

\section{MATERIAL}

Wir bevorzugen Procain 1\% ohne Zusatzstoffe (Lidocain kann alternativ verwendet werden). Procain hat eine durchblutungsfördernde Wirkung. Zudem erfolgt der Abbau im Gewebe (über die Pseudocholinesterase). Procain ist nebenwirkungsfrei und zeigt keine Interaktionen mit anderen Medikamenten, was beispielsweise in der geriatrischen (oft Polypharmazie) Schmerztherapie ein Vorteil ist. Daneben wirkt Procain unter anderem entzündungshemmend $^{6}$ und membranstabilisierend.

\section{INDIKATIONEN/KONTRAINDIKATIONEN}

Indikationen für eine Neuraltherapie sind akute und chronische Schmerzen, funktionelle Erkrankungen wie etwa Colon irritabile und Entzündungen (zum Beispiel adjuvant beim autoimmunen Formenkreis). Kontraindikationen sind Allergie gegen Lokalanästhetika (sehr selten bei Procain), Gerinnungsstörungen oder Antikoagulation sowie eine Tendenz zu vasovagalen Synkopen.

\section{KOMPLIKATIONEN}

Organverletzungen (Pneumothorax und andere) können auftreten. Versehentliche Injektionen in ein hirnwärts führendes Gefäß oder in den Liquorraum (Aspiration!) können Krämpfe, Atemstillstand, Bewusstlosigkeit, Herz- und Atemstillstand hervorrufen.

\section{NEUROPHYSIOLOGIE UND WIRKMECHANISMEN}

Das autonome Nervensystem ANS - insbesondere der Sympathikus - ist integriert bei Schmerz-Sensibilisierungsprozessen mit eventuell neuroplastischen Veränderungen, bei Immun- und Entzündungsprozessen und bei Mikrozirkulationsstörungen., 2, 4, 7-10 Die Regulation des Sympathikus durch einen passageren „Reset“ ist deshalb bei vielen Schmerz- und Krankheitsbildern von der Neurophysiologie aus gesehen logisch und nachhaltig, da sich das System danach selbst in Richtung eines physiologischeren Zustands organisieren kann (siehe Abbildung). Voraussetzung hierfür ist eine erhaltene Regulationsfähigkeit.

\section{WIRKSAMKEIT}

Aus der zunehmenden Anzahl an neuraltherapeutischen Publikationen in Pubmed möchte ich in diesem Rahmen zwei unserer umfangreichen Studien der Universität Bern (Dissertationen) diskutieren. Die erste Arbeit" ist eine vom schweizerischen Bundesamt für Gesundheit (BAG) unterstützte Cross-Sectional-Studie. Evaluiert wurden Patient*innen mit Erkrankungen des Bewegungsapparates in Grundversorger-Praxen mit und ohne Integration der Neuraltherapie. Bei allen Messgrößen wurden in der Neuraltherapiegruppe (NT) bessere Resultate erzielt im Vergleich zur rein konventionell-medizinischen Gruppe (COM).

Die zweite Studie analysiert 280 zwecks Neuraltherapie überwiesene Patient*innen mit chronischen Schmerzen und Therapieresistenz gegenüber konventionell-medizinischen Behandlungen. ${ }^{12}$ Bei 4/5 der Patient*innen konnte eine deutliche, anhaltende Besserung erzielt werden; davon wurden 15 Prozent schmerzfrei bei nur neun Konsultationen in einem Jahr. Bei 64 Prozent konnten die Schmerzmedikamente reduziert werden.

In einem „Antrag an das BAG "13 konnten wir zeigen, dass zwischen 2000 und 2008 pro Jahr und pro Patient*in sowohl die totalen als auch die Medikamentenkosten bei den COM-Grundversorgern signifikant höher waren als bei den NT-Grundversorgern.

\section{AUSBILDUNGSMÖGLICHKEITEN}

In verschiedenen Ländern existieren Ärztegesellschaften für Neuraltherapie mit ähnlicher Ausbildungsordnung unter 
Hier steht eine Anzeige.

Springer 
dem Dachverband IFMANT (International Federations of Medical Associations of Neural Therapy). In Österreich ist es die Österreichische Medizinische Gesellschaft für Neuraltherapie und Regulationsforschung. Empfohlene Lehrbücher: Barop $\mathrm{H}^{1}$, Fischer $\mathrm{L}^{2}$, Gold-Sklarski $\mathrm{K}^{3}$, Weinschenk $\mathrm{S}^{4}$.

\section{Referenzen:}

1 Barop H. Lehrbuch und Atlas der Neuraltherapie. Haug, Stuttgart 2016

2 Fischer L. Neuraltherapie. Neurophysiologie, Injektionstechnik und Therapievorschläge. 5. Aufl. Thieme, Stuttgart 2019

3 Gold-Szklarski K (Hrsg). Arbeitsbuch Neuraltherapie. Facultas, Wien 2009

4 Weinschenk (Hrsg). Handbuch Neuraltherapie. Thieme, Stuttgart 2020

5 Resch et al. Neuraltherapie. In: Gaul C und Diener HC (Hrsg). Kopfschmerzen: Pathophysiologie, Klinik, Diagnostik, Therapie. Thieme, Stuttgart 2016; 287-93

6 Cassuto $\mathrm{J}$ et al. Anti-inflammatory properties of local anesthetics and their present and potential clinical implications. Acta Anesthesiol Scand. 2006; 50(3):265-282

7 Elenkov IJ et al. The sympathetic nerve - an integrative interface between two supersystems: the brain and the immune system. Pharmacol Rev. 2000; 52(4):595-638

8 Jänig W, Baron R. Pathophysiologie des Schmerzes. In: Fischer L, Peuker E (Hrsg). Lehrbuch Integrative Schmerztherapie. Haug, Stuttgart 2011; 35-71

9 Schaible HG, Straub RH. Function of the sympathetic supply in acute and chronic experimental joint inflammation. Auton Neurosci. 2014; 182:55-64

10 Tracey KJ. The inflammatory reflex. Nature. 2002; 420(6917):853-859

11 Mermod et al. Patient satisfaction of primary care for musculoskeletal diseases: A comparison between Neural Therapy and conventional medicine. BMC Complement Altern Med. 2008; 8:33

12 Egli $S$ et al. Long-term results of therapeutic local anesthesia (neural therapy) in 280 referred refractory chronic pain patients. BMC Complement Altern Med. 2015; 15:200

13 Fischer L, Ludin SM, Thommen D, Hausammann R. Antrag auf Kostenübernahme durch die obligatorische Krankenpflegeversicherung betreffend der Störfeld-Therapie (Neuraltherapie nach Huneke) an das Schweizerische Bundesamt für Gesundheit. 2010

14 Fischer L. Physical principles - neurobiological principles. In: Liem T, van der Heede P (Hrsg). Foundations of morphodynamics in osteopathy. Handspring Publishing, Edinburgh 2017; 67-96

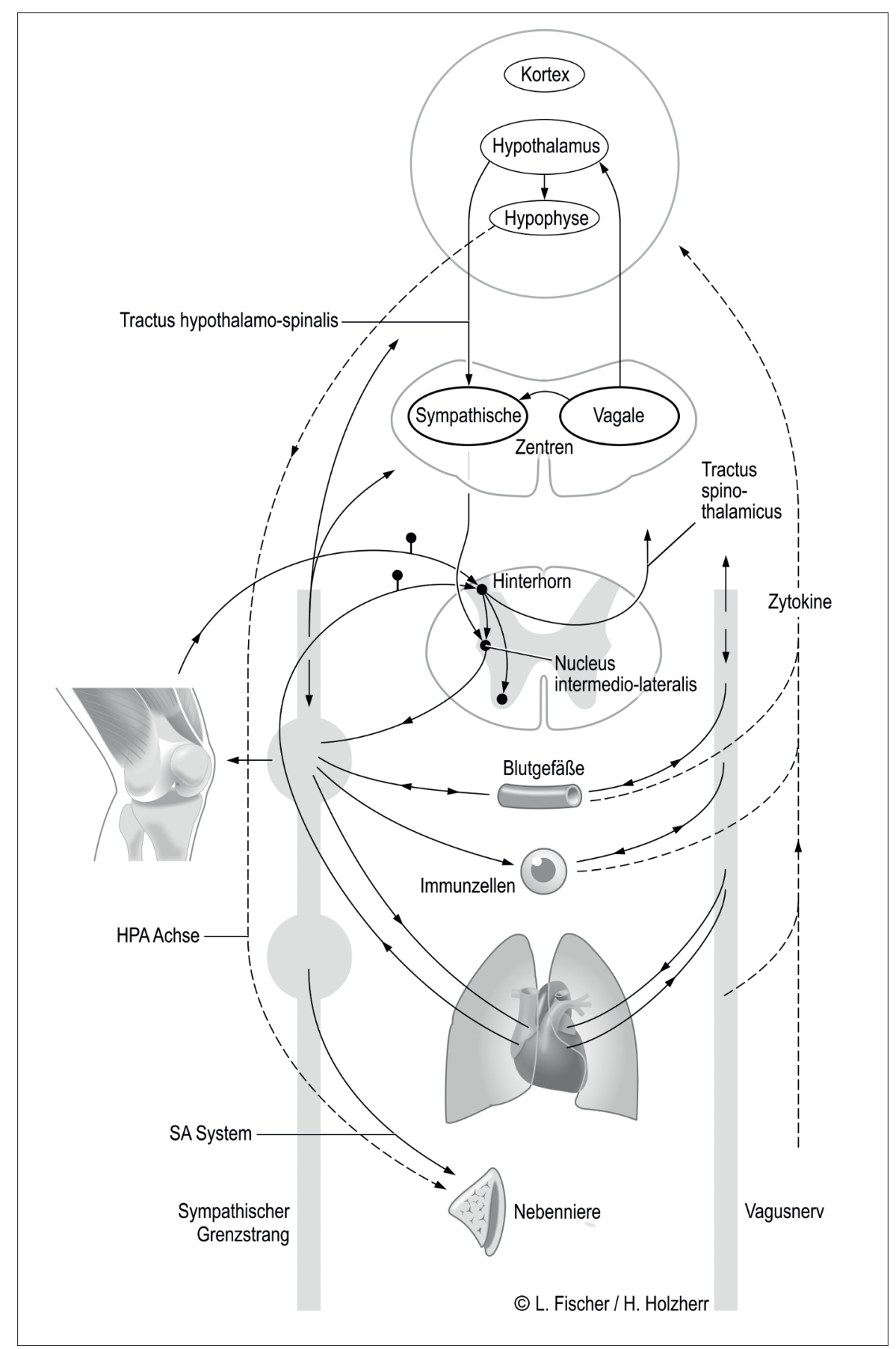

\section{Abbildung: Die Untrennbarkeit der Systeme (stark vereinfachte Darstellung)*}

Innere Organe, Haut, Muskulatur, Nervensystem und Interstitium (Immun- und Entzündungssystem, Mikrozirkulation) sind untrennbar untereinander verflochten. Hierbei übernimmt das ANS eine koordinierende Funktion. Dabei beteiligt es sich an vorwiegend positiv rückgekoppelten Reflexbögen, die die Peripherie mit den verschiedenen Ebenen des Zentralnervensystems eingeht: mit dem Rückenmark, dem Hirnstamm und dem Kortex. Dabei konvergieren Afferenzen aus der Peripherie auf dieselben Zellen im Hinterhorn. In der Folge werden sie in einem spinalen Reflexbogen zurück in die Peripherie geleitet, gleichzeitig aber auch über den Tractus spinothalamicus zum Gehirn. Sympathische und vagale Zentren im Hirnstamm komplettieren einen weiteren Reflexbogen, ebenso wie cranial Kortex, Hypothalamus und Hypophyse. Hierüber können auch
Emotionen modulierend Einfluss nehmen. Efferente sympathische Zentren im Hirnstamm erhalten also einerseits Informationen von vagalen Projektionskernen, andererseits vom Hypothalamus (Tractus hypothalamo-spinalis). So erhält der sympathische Ursprungskern im Rückenmark (Nucleus intermedio-lateralis) gleichzeitig Informationen von den Zentren im Gehirn und von der Peripherie. Die Komplexität wird noch erhöht, indem die Hypothalamus-Hypophysen-Achse (HPA) und das sympathisch-adrenale (SA) System regulierend eingreifen, ebenso wie die Zytokine. Hohe Komplexität und Rückkoppelungen zeigen die Untrennbarkeit der Systeme. Hohe Komplexität und Rückkoppelungen zeigen die Untrennbarkeit der Systeme. Für die Therapie ergeben sich verschiedene logische Angriffspunkte (siehe Injektionsarten). 